\title{
Effects of Different Hemodialysis Treatments on Abnormal Mineral and Bone Metabolism in Patients with Chronic Renal Failure
}

\author{
Qiang Li
}

Qingyun People's Hospital, Qingyun 253700, Shandong Province, China

\begin{abstract}
Objective: To investigate the effects of different hemodialysis treatments on abnormal mineral and bone metabolism in patients with chronic renal failure. Methods: A random number table was used to divide 80 patients with chronic renal failure admitted to our hospital from January 2018 to January 2019 into 2 groups, with 40 cases in each group. Group A was treated with low-flux hemodialysis, and group B was treated with high-flux hemodialysis. The related indicators of mineral and bone metabolism of the two groups were compared. Results: Before treatment, the blood calcium, blood phosphorus, intact parathyroid hormone (iPTH), type I procollagen amino terminal peptide (PINP), fibroblast growth factor 23 (FGF23), serum creatinine (Scr) indicators of the two groups were compared. The difference was not statistically significant $(P>0.05)$; After treatment, the blood calcium levels of the two groups were higher than before treatment, the blood phosphorus, iPTH, PINP, FGF23, and Scr levels were lower than before treatment, and the blood calcium level of group B was higher than that of group A, while blood phosphorus, iPTH, PINP, FGF23, and Scr levels were lower than group A, the difference was statistically significant $(P<0.05)$. Conclusion: Compared with low-flux hemodialysis, patients with chronic renal failure treated with highflux hemodialysis have better results, which can correct abnormal bone metabolism and improve Scr levels.
\end{abstract}

Keywords: Chronic renal failure; Hemodialysis; Abnormal mineral and bone metabolism

Publication date: November, 2020
Publication online: 30 November, 2020

*Corresponding author: Qiang Li, liqiang1977.0534 @163.com

Chronic renal failure is a serious kidney disease caused by a variety of factors. It has a high risk, and if it cannot be controlled in time and effectively, it is likely to cause serious threats to the life of patients. Hemodialysis is an effective way for the clinical treatment of chronic renal failure, and with the advancement of clinical medical technology, dialysisrelated complications are gradually reduced ${ }^{[1]}$. However, studies have pointed out that patients with chronic renal failure are those with abnormal mineral and bone metabolism, especially those who are treated with maintenance hemodialysis ${ }^{[2]}$. With the development of medical technology, there are various hemodialysis techniques for the treatment of this disease. Therefore, which dialysis method can reduce the abnormal bone metabolism of patients is the focus of clinical research. In view of this, this study aims to investigate the effects of different hemodialysis treatments on abnormal bone metabolism in patients with chronic renal failure. The report is as follows.

\section{Information and Methods}

\subsection{General Information}

Using random number table method, 80 patients with chronic renal failure admitted to our hospital from January 2018 to January 2019 were divided into 2 groups, each with 40 cases. Group A has 23 males and 17 females; ages are 25-76 years, with an average age of $(50.45 \pm 3.18)$ years; dialysis 
duration is 18-65 months, with an average duration of $(47.97 \pm 16.12)$ months. Group B has 24 males and 16 females; ages are 28-75 years old, with an average age of (50.16 \pm 3.54$)$ years; dialysis duration is $20-63$ months, with an average duration of $(47.58 \pm 16.23)$ months. The general data of the two groups were compared, and the difference was not significant $(P>$ 0.05 ), and the study was comparable. The study had been reviewed and approved by the medical ethics committee of our hospital and the informed consent form was voluntarily signed by the patients and their family.

\subsection{Inclusion criteria}

(1) Inclusion criteria: Hemodialysis duration is more than 12 months; Recent illness is stable; Estimated survival time $>1$ year. (2) Exclusion criteria: accompanied by blood system disease; combined with advanced tumor; severe mental illness; previously diagnosed as primary osteoporosis; undergoing parathyroidectomy.

\subsection{Methods}

\subsubsection{Group A}

Group A was given low-flux hemodialysis: F6 hemodialysis machine produced by Fresenius (Germany), polysulfone membrane, membrane area $1.30 \mathrm{~m}^{2}$, ultra-permeability $5.5 \mathrm{~mL} /(\mathrm{h} \bullet \mathrm{mmHg})$, once a week.

\subsubsection{Group B}

Group B was given high-throughput hemodialysis: Fresenius F6 hemodialysis machine, polysulfone membrane, membrane area $1.35 \mathrm{~m}^{2}$, ultra-permeability $34.0 \mathrm{~mL} /(\mathrm{h} \cdot \mathrm{mmHg})$, once a week.

Both groups of patients adjusted the frequency of dialysis according to urine output, and were given vitamin D (calcitriol) and calcium acetate. Both groups were treated continuously for 6 months.

\subsection{Evaluation Indicators}

The mineral and bone metabolism related indicators and renal function indicators of the two groups before treatment and 6 months after treatment were compared. $3 \mathrm{~mL}$ of venous blood from the patient was taken, and the serum was separated after centrifugation. The fully automatic biochemical analyzer was used to determine phosphorus, calcium and the intact parathyroid hormone (iPTH), type I procollagen amino terminal peptide (PINP), fibroblast growth factor 23 (FGF23), serum creatinine (Scr) levels.

\subsection{Statistical Methods}

SPSS22.0 software was used for data processing, measurement data was expressed in . Independent sample t-test was applied between groups and paired sample t-test was used within groups. $P<0.05$ indicates that the difference is statistically significant.

\section{Results}

Before treatment, the blood calcium, blood phosphorus, iPTH, PINP, FGF23, Scr indicators of the two groups were compared, the difference was not statistically significant $(P>0.05)$; after treatment, the blood calcium levels of the two groups were higher than before treatment, while the levels of blood phosphorus, iPTH, PINP, FGF23, and Scr of the two groups were lower than before treatment, and the blood calcium level of group B was higher than that of group A, while the blood phosphorus, iPTH, PINP, FGF23, and Scr levels of Group B were lower than group $\mathrm{A}$, the difference was statistically significant $(P<0.05)$. See Table 1.

Table 1. Comparison of related indicators of mineral and bone metabolism and renal function indicators between the two groups $(\bar{x} \pm s)$

\begin{tabular}{ccccc}
\hline Time & Group & Calcium $(\mathbf{m m o l} / \mathbf{L})$ & Phosphorous $(\mathbf{m m o l} / \mathbf{L})$ & $\mathbf{i P T H}(\mathbf{p g} / \mathbf{m L})$ \\
\hline Before Treatment & A $(n=40)$ & $2.22 \pm 0.21$ & $2.24 \pm 0.17$ & $410.07 \pm 28.45$ \\
& B $(n=40)$ & $2.23 \pm 0.27$ & $2.25 \pm 0.18$ & $409.85 \pm 28.62$ \\
& $t$ & 0.185 & 0.255 & 0.035 \\
After Treatment & $P$ & 0.854 & 0.799 & 0.973 \\
& A $(n=40)$ & $2.31 \pm 0.25^{\mathrm{a}}$ & $2.10 \pm 0.23^{\mathrm{a}}$ & $365.13 \pm 23.49^{\mathrm{a}}$ \\
& $\mathrm{B}(n=40)$ & $2.60 \pm 0.21^{\mathrm{a}}$ & $1.82 \pm 0.21^{\mathrm{a}}$ & $334.73 \pm 25.51^{\mathrm{a}}$ \\
& $t$ & 5.618 & 5.686 & 5.544 \\
\hline
\end{tabular}


Continue Table 1

\begin{tabular}{ccccc}
\hline Time & Group & PINP $(\mathbf{n g} / \mathbf{m L})$ & FGF23 $(\mathbf{n g} / \mathbf{m L})$ & Scr $(\boldsymbol{\mu m o l} / \mathbf{L})$ \\
\hline Before Treatment & A $(n=40)$ & $152.71 \pm 12.37$ & $428.09 \pm 32.38$ & $876.32 \pm 32.63$ \\
& B $(n=40)$ & $151.68 \pm 12.38$ & $428.06 \pm 31.39$ & $874.53 \pm 29.35$ \\
& $t$ & 0.372 & 0.004 & 0.258 \\
After Treatment & $P$ & 0.711 & 0.997 & 0.797 \\
& A $(n=40)$ & $146.68 \pm 11.47^{\mathrm{a}}$ & $395.58 \pm 30.36^{\mathrm{a}}$ & $485.63 \pm 28.63^{\mathrm{a}}$ \\
& $\mathrm{B}(n=40)$ & $133.67 \pm 10.45^{\mathrm{a}}$ & $367.85 \pm 29.33^{\mathrm{a}}$ & $401.59 \pm 26.33^{\mathrm{a}}$ \\
& $t$ & 5.303 & 4.155 & 13.665 \\
& $P$ & 0.000 & 0.000 & 0.000 \\
\hline
\end{tabular}

\section{Discussion}

As the patients' hemodialysis duration increases, the risk of fractures gradually increases, and studies have shown that the risk of fractures in hemodialysis patients is 17 times that of the normal population ${ }^{[3]}$. For example, if the blood phosphorus, blood calcium, iPTH, PINP, and FGF23 levels of patients exceed a certain range, it is closely related to the death of hemodialysis patients. Studies have pointed out that the blood phosphorus level of hemodialysis patients is only $39.6 \%$ that of normal level, while the blood calcium only accounts for $53.5 \%$, so it is of important clinical significance to improve the mineral and bone metabolism of hemodialysis patients ${ }^{[4]}$. The abnormality of mineral and bone metabolism in hemodialysis patients is mainly due to accelerated bone turnover, and the imbalance of osteoblast and osteoclast activity leads to abnormal mineralization and abnormal bone resorption, which affects the bone strength of patients and shows different degrees of osteoporosis and fibrous osteitis etc. FGF23 is a regulatory factor and its secretion level in the body increases with the increase in blood phosphorus, and the increase of FGF23 can down-regulate the expression of 1a-hydroxylase and reduce the synthesis of active vitamin D, leading to hyperthyroidism, which in turn promotes the secretion of iPTH in high level. PINP can reflect the ability of osteoblasts to synthesize bone collagen and bone turnover, and the increase in its level is closely related to the patient's bone density ${ }^{[5]}$. The results of this study showed that after treatment, blood calcium levels in the two groups were higher than before treatment, while blood phosphorus, iPTH, PINP, and FGF23 levels in the two groups were lower than before treatment, and blood calcium level in group B was higher than group A, while the levels of blood phosphorus, iPTH, PINP, FGF23, and Scr were lower than those of group A, indicating that compared with low-flux hemodialysis, adopting high-flux hemodialysis for patients with chronic renal failure can effectively improve the abnormal mineral and bone metabolism. The principle of high-flux hemodialysis includes adsorption, convection and diffusion, and the total calcium concentration is relatively stable, which can effectively enhance the activity of bone cells and promote the release of bone calcium. By reasonably using phosphorus binders and active vitamin D drugs, it can improve patients' blood calcium levels and related indicators of mineral and bone metabolism. Meanwhile, compared with low-flux dialysis, diafiltration can be added to high-flux hemodialysis on the basis of diffusion, which can effectively increase the clearance rate of iPTH and blood phosphorus. With the effective clearance of iPHT, FGF-23 level decreases, which inhibit the activity of osteoclasts, thereby reducing bone turnover and slowing down bone resorption ${ }^{[6]}$. However, due to the small number of selected cases in this study and the limited follow-up time, a larger sample size should be used in future studies with extended follow-up time to further study the effects of high-throughput hemodialysis on patients with chronic renal failure.

In conclusion, high-flux hemodialysis can effectively rectify the abnormal mineral and bone metabolism in patients with chronic renal failure, improve renal function, and is worthy of clinical promotion.

\section{References}

[1] Wang Y, Xue YH. Effect of intravenous calcitriol on bone metabolic markers in patients with chronic renal failure complicated with mineral and bone abnormalities [J]. Journal of North Sichuan Medical College, 2019, 34(6): 760-762, 771.

[2] Tan HJ, Ge L, Liu NL, et al. Hemodialysis Combined with Low Frequency Hemoperfusion in the Treatment of Abnormal Mineral and Bone Metabolism in Patients with Chronic 
Kidney Disease [J]. Chinese Journal of Microcirculation, 2019, 29(1): 81-84.

[3] Chen YR. The effect of hemoperfusion combined with hemodialysis on blood phosphorus immunoreactive thyroid hormone levels in patients with chronic kidney disease and bone abnormalities [J]. Shanxi Medical Journal, 2018, 47(15): 77-79.

[4] Chen YR. The effect of hemoperfusion combined with hemodialysis on blood phosphorus immunoreactive thyroid hormone levels in patients with chronic kidney disease and bone abnormalities [J]. Shanxi Medical Journal, 2018, 45(15):
1820-1822.

[5] Li ZH, Li ZK, Zhang RR. A comparative study of different active vitamin $\mathrm{D}$ in the treatment of chronic kidney disease minerals and bone abnormalities in maintenance hemodialysis patients [J]. Shaanxi Medical Journal, 2017, 46(5): 656-658.

[6] He GF, Chen WL, Lai YH, et al. Treatment effectiveness of different blood purification modes for mineral and bone metabolism disorder in patients with maintenance hemodialysis[J]. Journal of Clinical Nephrology, 2019, 19(6): 453-455. 